\title{
Using sequential synthesis problems to investigate novice teachers' conceptions of hydrodynamics
}

\author{
Zehao Jia $\odot,{ }^{1}$ Lin Ding $\odot{ }^{2}$ and Ping Zhang ${ }^{1, *}$ \\ ${ }^{1}$ Department of Physics, Beijing Normal University, Beijing 100875, People's Republic of China \\ ${ }^{2}$ Department of Teaching and Learning, The Ohio State University, Columbus, Ohio 43210, USA
}

(Received 4 November 2020; accepted 3 May 2021; published 21 June 2021)

\begin{abstract}
We used a sequential synthesis problem to investigate novice teachers' conceptual difficulties in hydrodynamics. Twenty-one new secondary level physics teachers from various regions of China, who had been in service for no more than 2 years, participated in the study. Each participant completed a written hydrodynamics problem consisting of four questions, all of which were related to a situation about water flowing in a long, nonuniform tube of different cross sections, heights, and orientations. Analysis of the teachers' written performances exposed a number of errors. To further investigate their underlying notions about hydrodynamics, we conducted one-on-one interviews with 13 teachers selected randomly from those making such errors on the written test. The interviews revealed three major categories of errors about hydrodynamics held by our novice teachers. These relate to (a) ontological confusions and misuse of properties associated with solid, liquid, and gas; (b) deficient perceptions of force-motion and work-energy analysis; and (c) mischaracterizations about the nature of Bernoulli's equation and the quantities therein. Here, we not only replicated some of the literature-reported misconceptions but also uncovered new ontological notions of hydrodynamics held by physics teachers, an underresearched population on this topic.
\end{abstract}

DOI: 10.1103/PhysRevPhysEducRes.17.010142

\section{INTRODUCTION}

Hydrodynamic applications are ubiquitous [1] and have direct and significant impacts on our everyday lives. It is no surprise that the science content standards for secondary physics in China include fluid concepts, such as pressure and buoyancy [2], as part of the key ideas to be discussed in class. This requires physics teachers to have robust understandings of various topics in fluid mechanics. To that end, fluid dynamics has traditionally been an integral component of the "Special Topics in Fundamental Physics," a professional development course that we designed and taught for new, in-service secondary physics teachers as part of their continuing education program.

Conceptual difficulty is one of the earliest and most widely studied areas in physics education research $[3,4]$. Research on this topic has uncovered a collection of alternative ideas held by science learners and has informed the design and implementation of various curricula, including those for teacher education and training. Although

\footnotetext{
*To whom correspondence should be addressed. zhangping@bnu.edu.cn

Published by the American Physical Society under the terms of the Creative Commons Attribution 4.0 International license. Further distribution of this work must maintain attribution to the author(s) and the published article's title, journal citation, and DOI.
}

many misconceptions have been documented for both introductory and advanced-level physics, the task of creating a complete catalog of alternative conceptions in all topics is still daunting [3]. In the area of hydrostatics, there have been studies of student conceptual difficulties with buoyancy, pressure, and other related topics, such as Archimedes' principle [5-15]. However, research on misconceptions about hydrodynamics is still scant. Of the few studies on this subject, nearly all have been limited to discussions about the challenges in teaching Bernoulli's equation or development of assessment instruments such as the Fluid Mechanics Concept Inventory (FMCI) [16-30]. In addition, the target populations of the prior studies have mostly been students. Physics teachers, on the other hand, have by far received disproportionally little attention on this matter.

In this study, we used a nontypical approach to investigate teachers' notions of hydrodynamics. Specifically, we created a sequential synthesis problem in which the teacher participants needed to reason through a set of sequenced phenomena or events by integrating multiple hydrodynamics concepts and employing different representations, such as diagrams and graphs. This allowed us to examine the teachers' notions about hydrodynamics in concrete scenarios. More importantly, our use of a sequential synthesis task allowed us to uncover what otherwise would have been difficult to capture had we used a set of individual, unrelated single-concept questions. Here, our goal is to 
understand how novice teachers perform hydrodynamics tasks. More specifically, we attempt to answer the following questions.

(i) What common errors do novice teachers demonstrate in solving a sequential synthesis problem involving an ideal fluid flow under a sequence of different conditions?

(ii) What underlying notions do novice teachers hold about hydrodynamics that may have contributed to their errors?

\section{PRIOR STUDIES AND THEORETICAL BASIS}

\section{A. Misconceptions in fluid mechanics}

Prior research on teaching and learning of hydrodynamics has revealed several common misconceptions held by students. One pertains to their understandings of the incompressibility of liquid. (Note that unless otherwise indicated, the terms of fluid and ideal fluid henceforward refer to liquid only.) In the context of hydrostatics, Kariotoglou et al. [10] and Kariotogloy et al. [11] found that students entirely overlooked the incompressibility feature and thought pressure in fluid would increase when the fluid was confined in a smaller place. In teaching hydrodynamics, Suarez et al. [31] and Brown et al. [32] also found that when answering questions about water flowing vertically downward out of a tube or into a narrower section of a horizontal pipe, students often used a smaller cross section as evidence to argue that water was compressible and hence reasoned that water pressure would increase after it entered a constricted region. Additionally, despite the different mechanisms for an increased flow speed in the above two scenarios, students invariably drew on the idea of water being compressible to conclude that speed and pressure would both increase as the cross section of the flow decreased. Similar findings were also reported by Watson et al. [18] who examined students' performances on the FMCI questions.

Learners' conceptual difficulties with force analysis are another important part of the previous studies. In hydrostatics, Kariotoglou et al. [10] found that students directly related fluid pressure to the weight placed above it. Similarly, as Besson [6] mentioned, students only included into analysis forces from above a fluid element but ignored those from underneath it. This error, as Suarez et al. [31] noted, was also detected in the context of hydrodynamics, where students considered upstream of a fluid to be the sole source of external force on a fluid element and applied it to both confined and unconfined fluids.

When it comes to the relationship between pressure and speed in hydrodynamics, students often invoked the idea of "the greater speed the less pressure." This, as Smith [22] mentioned, was what many physics teachers would refer to as a quick summary of Bernoulli's equation; but it left out important details of the conditions under which this idea might apply. Here, it is important to note that Bernoulli's equation is derived from the workenergy theorem under the assumptions that an incompressible fluid undergoes a steady, laminar flow with no friction, and that the principle is to be applied to fluid elements along the same streamline [33-36]. However, even after learning this principle, students still failed to invoke it for problem solving. For example, Suarez et al. [31] found that in the context of water flowing vertically down a uniform tube, many students either disregarded the change in water pressure and treated the situation as a simple free fall, or ignored water speed and equated the situation to a hydrostatic case by concluding pressure to be in direct proportion to height. Even for those who did invoke Bernoulli's equation, they showed various difficulties with its application. For instance, Recktenwald et al. [37] found that students improperly applied Bernoulli's equation to a nonsteady, turbulent flow in a region where there is a noncontinuous change in cross section along a pipe. Similarly, Suarez et al. [31] found that students often mechanically applied Bernoulli's equation and neglected the height of a flow by concluding a direct relationship between an increased speed and a decreased pressure.

\section{B. Uncovering and understanding misconceptions}

In studies of misconceptions, researchers have proposed different frameworks to account for students' conceptual difficulties. For example, Chi's ontological category framework describes students' robust misconceptions as a result of their miscategorization of ontologically different existences [38,39]. According to Chi, students may mistakenly assign characteristics possessed exclusively by one category of existence, such as entities, to another category, such as processes. A corollary is that students confuse between different ontological existences and form misconceptions that are resistant to change. While Chi's reference to ontology was at the highest level of hierarchy (meaning no shared superordinate-level category between any two ontologies), other researchers have applied this idea to lower levels. For example, Hoehn, Gifford, and Finkelstein [40] described wave and particle as two distinct ontologies, although both could be fitted under Chi's "entities" category. Similarly, Scherr and colleagues $[41,42]$ identified three ontologies of energy used by students and physicists, namely, substance, stimulus, and vertical location, while in fact these ontologies can also be subsumed under Chi's "entities" category. In the current study, we align our use of the term "ontology" to those in the studies by Hoehn et al. and Scherr et al. and apply this approach to investigate participants' ideas of fluid dynamics. Simply put, we consider categories at lower hierarchy, for example, different states of matter as 
distinct ontologies ${ }^{1}$ since they each exclusively possess properties that others do not. In addition to Chi's ontology framework, Vosniadou $[43,44]$ proposed a "theory" theory to account for learners' intuitive ideas, particularly those of young children's. She argued that their conceptions originated from relatively coherent, frameworklike "theories"; hence, children can use incorrect ideas in a consistent manner to make explanations and predictions of natural phenomena. In contrast, DiSessa's knowledgein-pieces theory highlighted the fragmented nature of learners' intuitive ideas and contended that these ideas were made of $p$ prims and were not coherently organized [45]. Drawing on this notion, Hammer and colleagues further proposed a resource framework, in which they conceptualized learners' knowledge structure as a system of loosely connected resources, capable of being activated or deactivated depending on the contexts [46].

As Brown et al. [32] pointed out, context plays a significant role in students' thinking, and students' misconceptions are often situational. For example, with everything else being the same, after a tube was raised vertically up from its initially horizontal position, student approaches to the otherwise isomorphic question differed drastically. Therefore, it is important to design scenarios in which student misconceptions can be fully exposed and clearly identified. Brown et al. [32] also found that when responding to multiple-choice questions, some participants wished to select an answer that was not provided in the given choices, and yet other participants did not believe any of the given options was correct but felt forced to select one.

To mitigate the constraints placed on students' thinking due to question types and the ways questions are phrased, multiple representations can be employed as an effective solution. Studies have shown that student performance on physics problems varies with representational formats [47]. Kohl et al. [48] found that students generally viewed mathematical expressions and diagrams as two primary, but mutually exclusive, representational tools in physics. They associated diagrams strongly with physics concepts but could not see the inherent connections between mathematics and physics concepts. To that end, requiring students to use different representation formats to tackle physics tasks can be an effective way to reveal their hidden conceptions. This point was clearly illustrated in Cao's study of high school students' understandings of electric fields, in which diagrammatic representations were shown

\footnotetext{
${ }^{1}$ Perhaps we may call them ontological branches to better align to Chi's use of the term "ontology". It is worth noting that we do not intend to engage in a philosophical debate of what types of existence deserve the name ontology, as it is beyond the scope of the study. We do, however, propose the aforementioned use of this term for the convenience of conveying findings. In fact, even in her seminal paper, Chi [39] put forth a disclaimer stating that her definition of ontology was not meant to be conclusive.
}

to be a powerful tool to uncover learners' hidden conceptions [49].

In review of the prior work on teaching and learning fluid mechanics $[18,28,31,32]$, we found the tasks used were all short questions of independent scenarios. Differing from this convention, we created and used a sequential synthesis problem [50-55] to study novice teachers' understandings of hydrodynamics (see Sec III. for details). Here, the problem involves multiple phenomena taking place in a chronological order. It depicts a water flow undergoing a sequence of changes due to varying conditions. In a sense, each phenomenon represents a scenario of similar hydrodynamics concepts but is designed to be in differing surface features. A successful problem solver needs to jointly apply multiple hydrodynamics concepts consistently to capture these sequenced events.

\section{METHODOLOGY}

\section{A. Participants}

The participants of the study were a cohort of 21 novice teachers who had earned a bachelor's degree in science from the physics department at a large research university in China. By the time of the study, they had only taught physics full-time for 2 years in their respective secondary schools. All of the participants had systematically studied physics during their undergraduate program.

This study took place in a continuing professional development course in summer 2019, titled Special Topics in Fundamental Physics. Designed specifically for new in-service teachers, this course integrated university physics and secondary-level pedagogies and was organized into a series of topical content areas. Each class consisted of two parts, a lecture on physics content followed by open discussions among the participating teachers to solidify their understandings and applications of learned knowledge. Four hours of class time were devoted to fluid dynamics, including topics of the continuity equation, Bernoulli's equation and assumptions for ideal fluids. These concepts were all part of the universitylevel introductory mechanics that the novice teachers had taken six years ago in their freshmen year. In the present study, the teachers revisited fluid dynamics in class before taking a written test (see below). Scores on the test did not count toward their final course grades, but we encouraged them to take the test seriously by asking them to give their best and honest answers.

\section{B. Tasks and analysis}

We designed a sequential synthesis problem, in which an ideal incompressible fluid (water) flows through a nonuniform tube of variable cross sections, heights, and orientations and finally runs out of the tube. This problem requires our participants to invoke multiple hydrodynamics concepts, including fluid element, speed, pressure, and energy 


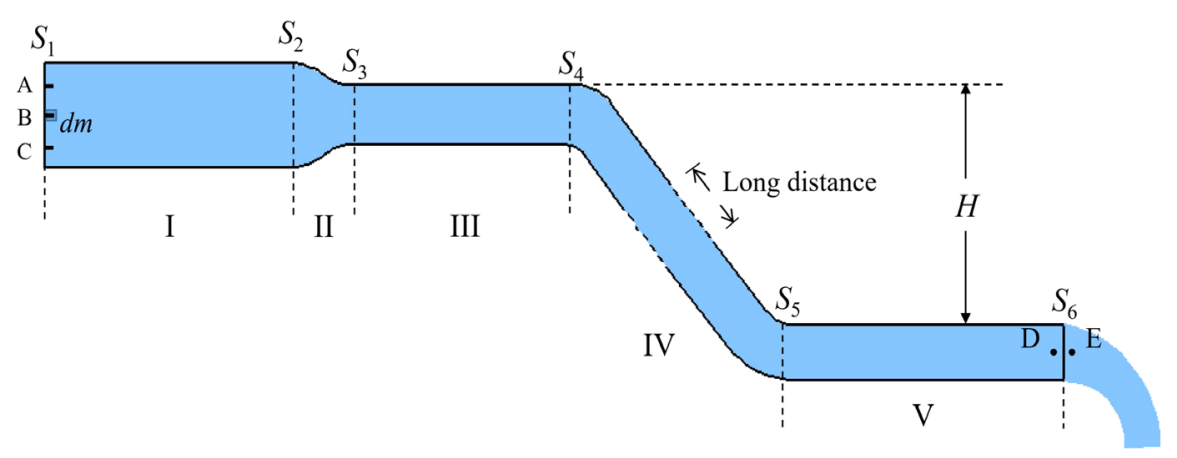

FIG. 1. Water flows in a nonuniform tube of different cross sections and heights. It enters at $S_{1}$ and exits to open air at $S_{6}$

in relation to Bernoulli's equation and the continuity equation. It is worth noting that the problem contains a set of sequenced phenomena or events, each representing a spatiotemporally separate scenario. It differs from a traditional multipart problem where a single phenomenon or event is conceptually divided into a set of operationally manageable steps, each serving as scaffolding to bring a problem solver progressively closer to the final answer.

The statement of the test problem is quoted verbatim as follows. Consider a small and smooth tube with a circular cross section all along, as shown in Fig. 1. Six cross sections $S_{1}-S_{6}$ and five regions $\mathrm{I}-\mathrm{V}$ are marked in the diagram. The cross section of the tube decreases gradually throughout region II but remains constant in regions III-V. Regions I-III of the tube are leveled at the same height (note that diameter is negligible relative to height). Beginning in region IV, the tube gradually decreases in height until in region $\mathrm{V}$, where it stays leveled again. Water enters the tube from the left of $S_{1}$ with an initial horizontal speed $v_{1}$ and pressure $P_{1}$, and finally it flows out of the tube into open air at $S_{6}$. Consider the water as an ideal fluid undergoing a steady streamline flow in the entire process.

We asked our teachers to answer the following questions.

Q1. Marked on $S_{1}$ are the beginning segments of three streamlines of the flow at points $A, B$, and $C$, respectively. Please complete the streamlines in all regions along the tube from $S_{1}$ to $S_{6}$.

Q2. Select a water element $d m$ at point $B$, the inlet of the tube. Discuss the change in its speed $v$ and pressure $P$ along the tube from $S_{1}$ to $S_{6}$, respectively.

Q3. $D$ and $E$ are two locations near the outlet $S_{6}$ of the tube. $D$ is at the inside of the tube and $E$ is at the outside. Please compare the speed and pressure of the water at $D$ with those at $E$, respectively. Also compare the water pressure at these two points with the atmospheric pressure $P_{0}$, respectively.

Q4. Fig. 2 shows a coordinate system. The $x$ axis represents the horizontal position along the tube, and the $y$ axis represents three quantities $\frac{1}{2} \rho v^{2}, \rho g h$, and $P$ of a water element $d m$. The initial values of the three quantities at $S_{1}$ are marked on the $y$ axis. Please draw three curves, representing $\frac{1}{2} \rho v^{2}, \rho g h$, and $P$, respectively, as a function of $x$, as $d m$ moves from $S_{1}$ to $S_{6}$.

This sequential synthesis problem required our participants to invoke properties of ideal fluid in conjunction with Bernoulli's equation to identify key quantities of a water system at various points along a tube. To be successful in solving the problem, participants must understand the difference between streamline and path line, the difference between static pressure and stagnation pressure, and the fundamental assumptions for Bernoulli's equation that relate directly to the properties of ideal fluid. These concepts, while basic in introductory fluid dynamics, are often challenging for learners and even physics instructors. To assist readers in better understanding these concepts in the context of solving the synthesis problem, we include correct answers in the Appendix. Also included therein is a list of relevant core ideas in introductory fluid dynamics that are required of university physics majors in China.

We administered the sequential problem to the teacher participants as a 30-min written test in class.

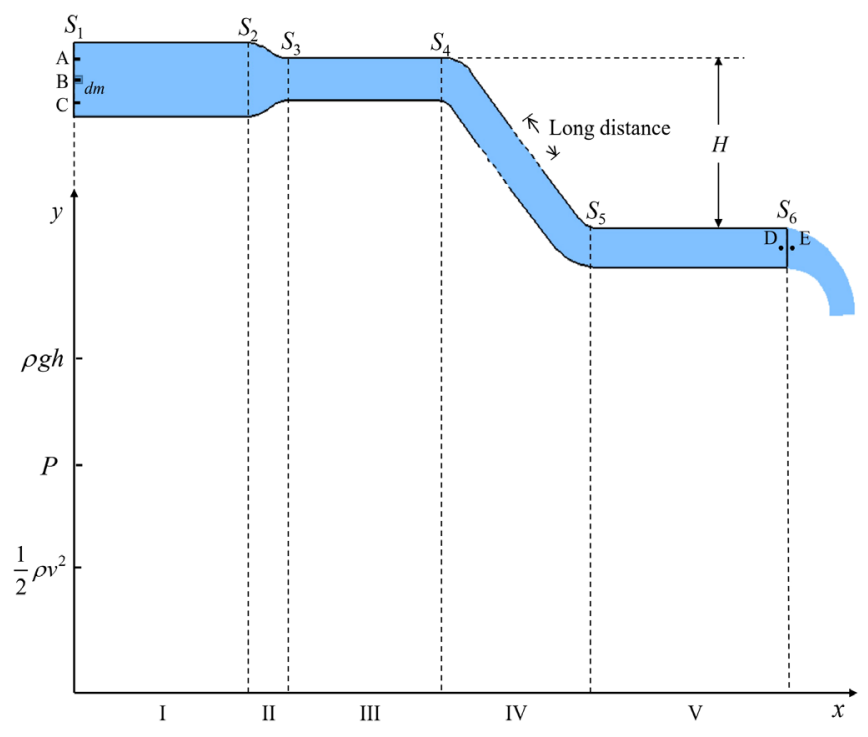

FIG. 2. A coordinate system for graphs of three quantities $\frac{1}{2} \rho v^{2}$, $\rho g h$, and $P$ as a function of the horizontal position $x$ of a fluid element. 
TABLE I. Error patterns, percentages of error, and interviewee identifiers for each sequenced task of the hydrodynamics problem.

\begin{tabular}{lccc}
\hline \hline Task & Patterns of error & Number $(\%)$ & Interviewees \\
\hline Q1 & Combine streamlines in narrow regions & $6(29 \%)$ & $\mathrm{T} 1, \mathrm{~T} 5, \mathrm{~T} 8$ \\
Q2 & $P$ decreases across region I & $3(14 \%)$ & $\mathrm{T} 3, \mathrm{~T} 16, \mathrm{~T} 18$ \\
& $P$ remains constant in region IV & $7(33 \%)$ & $\mathrm{T} 6, \mathrm{~T} 9, \mathrm{~T} 10$ \\
Q3 & $P_{D}=P_{E}>P_{0}$ & $10(48 \%)$ & $\mathrm{T} 1, \mathrm{~T} 7, \mathrm{~T} 11$ \\
& $P_{D}=P_{E}<P_{0}$ & $2(10 \%)$ & $\mathrm{T} 2, \mathrm{~T} 8$ \\
Q4 & Add a delay between $P$ and $\frac{1}{2} \rho v^{2}$ & $2(10 \%)$ & $\mathrm{T} 11, \mathrm{~T} 15$ \\
\hline \hline
\end{tabular}

Their responses were collected and analyzed. We first identified patterns of error revealed from the participants' responses. Then, for each of the identified pattern, we randomly selected 2-3 participants who made such errors for one-on-one semistructured interviews. Each interview lasted for 15-20 min, during which the teacher participant was required to explain their written responses in detail. To minimize unnecessary interventions, the interviewer would only encourage the participant to continue talking when there was a long pause or would ask the participant clarifying questions to better understand their ideas. All interviews were initially conducted, transcribed, and analyzed in Chinese before being translated into English. The authors independently studied the transcripts and the original videos to highlight the representative excerpts indicating the teachers' alternative ideas. From these excerpts, we then iteratively grouped the identified ideas and excerpts into categories that could capture the most foundational notions held by the participants about hydrodynamics. In the course of the work, we regularly compared notes among the authors and discussed any exisiting differences until a full agreement was reached. In what follows, we report the identified patterns of error and the teachers' notions about hydrodynamics.

\section{ANALYSIS AND FINDINGS}

\section{A. Written results}

From the participants' written responses, we identified their patterns of error in each question of the hydrodynamics problem. These patterns speak directly to our teachers' faulty notions of fluid streamline, pressure, and Bernoulli's equation. A major error emerging from question 1 is that the teachers treated streamlines as something that ought to be merged as the water entered into narrow regions of the tube. In questions 2 and 3, what stands out is the teachers' difficulties with water pressure under various conditions. They considered what should be a constant water pressure across region I as decreasing and what should be an increasing water pressure across region IV as constant. Also prominent is their difficulty in dealing with pressure at the interface between water and the atmosphere. As for graphing the different variables along the tube in question 4, some teachers treated these variables as not in sync with each other but instead added a delay between $P$ and $\frac{1}{2} \rho v^{2}$. Table I shows these error patterns and the percentages of the participants who made such errors. Also shown in Table I are the identifiers of those who participated in interviews (see Sec. IV B for interview results).

\section{B. Interview results}

Behind these error patterns underlay some of the most fundamental notions about the nature of fluid held by our participants. We uncovered a number of these notions from the interviews and summarized them into three categories related specifically to (a) indiscriminate use of features inherent to ideal liquid, solid, and gas models; (b) improper analysis of fluid element; and (c) misinterpretation of the nature of Bernoulli's equation. In what follows, we discuss each of the three categories with illustrations from our participant interviews.

\section{Ideal liquid, solid, and gas models}

Many of the participants' errors rooted deeply in their ontological confusions about the properties of ideal fluids. Our teachers frequently treated liquid as either a solidlike or a gaslike substance, conflating the key features of three commonly used models of solid, liquid, and gas.

Treat water (ideal fluid) as a solidlike substance. When discussing ideal fluid, some of the teachers correctly mentioned its incompressibility feature but unfortunately equated incompressibility to zero deformation. In other words, they considered each water element either as a rigid body where the distance between any two points would remain constant (cf. solid model) or as small particles with negligible volume (cf. simplified solid model). As a result, they conceptualized ideal fluid as a collection of nondeformable "cubes" or point particles. This notion of ideal fluid has not been previously reported in the literature but was captured in our teacher interviews. For example, one teacher participant said

T1: Water is incompressible, and the fluid element is so small that it can be seen as a cube. There is not enough space to enter the narrow tube, so you have to let it go through it one at a time......Umm, like many people entering the same door, they can only pass through it one by one. 


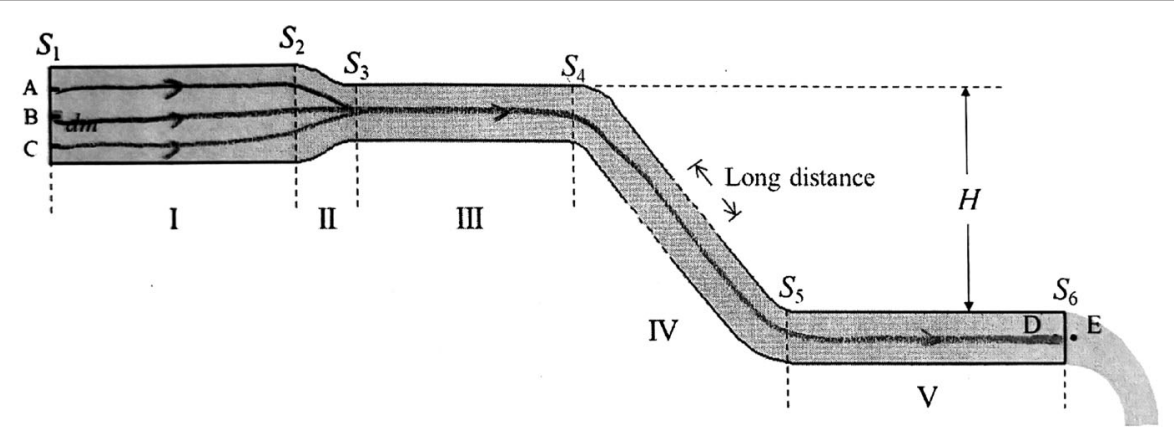

FIG. 3. A drawing of streamlines by a teacher participant (T1).

Another teacher participant used ideas derived from the point-particle model to treat water and said

T8: Well, if a fluid is incompressible, you can think of a fluid element as a point particle. It's like when you think of the earth revolving around the sun, the earth can be seen as a point particle. Like sand in an hourglass, they flow through narrow surface one by one.

Because of their use of solid-model-like features to conceptualize water, these participants came to such a mental picture that there would be fewer water elements passing through a narrower cross section at a time. Or put differently, they considered the instantaneous number of water elements on a cross section to be proportional to the area. That is why they merged the initial three streamlines into a single streamline as the water entered region II of a smaller cross section (see Fig. 3). In principle, streamlines are curves that are tangential to the velocity vectors of fluid elements, and their density is proportional to the magnitude of the local velocity. In other words, streamlines are diagrammatic representations of a velocity field. However, our participating teachers mischaracterized these representations as a trajectory of small solids. As a result, their streamlines would merge or split as a function of cross-sectional area.

Treat water (ideal fluid) as a gas-like substance. Contrary to the above cases, other participants invoked what should have been the properties of gas to reason about water. One particular type of ontological error in this category is to think of ideal fluid (water) as compressible like gas compression. For example, when explaining why he merged three streamlines into one at a narrower cross section, one teacher (T5) stated

T5: Water is a fluid. As the tube narrows, there is not enough space, and the fluid elements will be compressed together to pass through the tube. The density of the fluid increases after compression. Three fluid elements, each with a unit volume, now are being compressed into one unit. The fluid elements on the three streamlines become one when the tube narrows, so there should be only one streamline.
Clearly, the participant treated water as compressible like gas. In his opinion, a decrease in cross section would result in water compressing and thus an increased density. As with the above cases, the participant drew what seemed to be the trajectories (path lines) of water elements to represent streamlines and combined the initial three lines into one at a narrower section of the tube. Despite the same drawings, the underlying mechanism here is fundamentally different from that of the above. Here, the participant conceptualized water as a compressible substance like a gas and thereby concluded a reduced number of water elements after compression, whereas in the above cases the participants conceptualized water to be made of small nondeformable solids such that only a fewer number of them could pass through a narrower cross section at a time (and hence fewer streamlines).

Another type of reasoning under this category, which also has not been reported in the previous literature, is to treat water as a gaslike freely moving substance by neglecting cohesion in liquids. For instance, two interviewees provided the following rationale to conclude that the static pressure in water at the outlet of the tube would be less than the atmospheric pressure:

T2: At point E, I'm sure...its pressure is less than atmospheric pressure, otherwise the water flow will disperse, it is atmospheric pressure that makes the water gather to be a column.

T8: At point E, I think it should be lower than the atmospheric pressure because... I think water is bound by atmospheric pressure and becomes a column. If there is no atmospheric pressure and there is no external constraint, the water will disperse like gas.

Here, both teachers borrowed features charactieristic of a gas (cf. gas model) to account for water. They thought water particles would diffuse the same way as gas molecules if the atmospheric pressure were not sufficiently great.

Additionally, some teachers confused the mechanism of water pressure with that of gas pressure. Specifically, they invoked the concept of density as the sole determinant to 
analyze both water pressure near the outlet of the tube and the atmospheric pressure. To the best of our knowledge, this notion of pressure mechanisms has not been documented in the previous studies but emerged in our study. Below is such an interview except between a participating teacher and the interviewer.

T11: Compared to $P_{0} \ldots$ it is obvious that the atmospheric pressure is not as big as that of D and E because the liquid pressure is definitely greater than the gas pressure. It's a common sense.

Interviewer: You say it's a common sense... Can you explain what you are thinking?

T11: That is, umm, under the same volume, the greater the density, the greater the pressure. The density of the liquid must be greater than that of the gas, that's what I thought... So, the liquid molecules are pressed so close together. Think of the molecules of the air, far apart.

As seen, the participant resorted to the idea of intermolecular distance to conceptualize the same mechanism for both water and air. It is true that for a closed system of gas, reducing intermolecular distance can lead to increased density and pressure (an increased number of collision on the walls of a container in a certain time), but the mechanism for water pressure is fundamentally different. However, the participant indiscriminately used a mechanism that would only be suitable for ideal gas to account for water pressure.

\section{Analysis of fluid elements}

Also prominent from our interviews are the teachers' struggles with the analysis of fluid elements, a topic that has not been well studied in the area of hydrodynamics. Choosing a proper system of fluid element and separating it from its surroundings for force-motion and work-energy analysis is critical in reasoning about hydrodynamics. However, our participants revealed noticeable deficiencies in this area.

Defective performance on force-motion and work-energy analysis. While our teacher interviewees attempted to perform force-motion and work-energy analysis to answer hydrodynamic questions, their deficiency in carrying out some of the most basic evaluations, such as force analysis, was a major roadblock. For example, when responding to the question of fluid speed and pressure in Q2, one teacher drew three juxtaposed fluid elements $A, B$, and $C$ near the borderline of regions I and II with elements $A$ and $B$ in region I and element $\mathrm{C}$ in region II (see Fig. 4). He then continued

T3: For example, these three elements are on the same streamline [Fig. 4]. The kinetic energy of elements A and $B$ on the left side remains constant [marking " $E_{k}-$ "], but the kinetic energy of the fluid element $C$

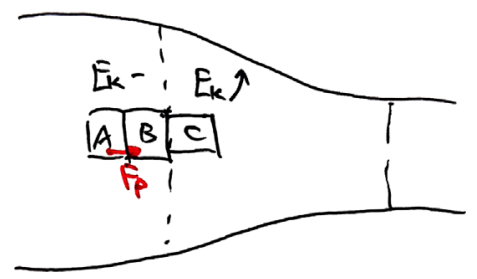

FIG. 4. A force diagram, drawn by a teacher participant (T3), of fluid elements at the borderline of regions I and II.

on the right side begins to increase [marking " $E_{k} \uparrow$ ”]. Where does the energy come from?... Element A exerts a force on element $B$ to do positive work on it, while A keeps its own kinetic energy unchanged. So, A should receive an external force from the left. I marked this force as $F_{P}$. ... Um, I remember our middle school textbook mentioned that a pressure difference between the upper and lower surfaces of a cube immersed in water can bring an upward buoyancy. Here, the pressure difference causes $F_{P}$, and the pressure on the left side of $A$ is greater than that on the right side.

After region II, the cross section of the tube no longer changes. The kinetic energy of a fluid element will no longer accelerate, so water pressure will not change in region III.

Here, the participant began with a focus on the kinetic energy of element $A$ and correctly recognized an external force that $A$ received from the left. However, what he failed to realize was that this was not the only horizontal force on element $A$. As such, he mistakenly took this individual force as a net force on $A$ to conclude a pressure difference. His subsequent reference to buoyancy was to reassure himself of the "correctness" of his conclusion and also to close the gap in his explanation by relating force to pressure. Interestingly enough, the same participant then continued to analyze region III and said that the cross section of the tube from region III and afterwards "no longer changes," therefore the kinetic energy of a fluid element and "water pressure will not change in region III." In other words, he used the downstream cross section being invariant and equal to that in region III as the sole determinant to conclude about the motion and pressure of the water in region III, skipping the force-motion analysis entirely.

Another teacher followed a similar line of reasoning to answer Q2. Differing from the above participant who mostly resorted to a force analysis to tackle the problem, this teacher attempted to use the work-energy relationship to answer the question but failed to account for the total work on the system of choice. Below is an interview excerpt of his analysis of water pressure in region I. Note that the teacher used region II, the downstream, as a point of departure to infer water pressure in region I.

T18: The flow speed in region II increases, so energy is needed. Energy comes from the inlet of the tube on the 
left... Without first doing work on the fluid near the inlet in region I, how would the energy be transmitted to the distal region II? Doing positive work on the fluid element in region I means that there is a net force to the right, so the pressure decreases.

As seen, the teacher created a fictitious agent, external to the water in the tube, as an energy source to supply kinetic energy to the water. He attempted to use the causal relationship inherent in the work-energy theorem to infer a positive work done on the system (water in Region I) by the external source, but he failed to take into consideration the negative work done by the downstream water on the right (perhaps because he viewed the water system to be a mere transmitter of energy from the upstream to the downstream). Ultimately, he used what in fact should be an individual work in place of the total work to conclude a nonzero net force on the system, hence reaching an incorrect answer about water pressure.

\section{The nature of Bernoulli's equation}

Besides the above findings, we also uncovered areas related to the teachers' notions about the nature of Bernoulli's equation. Specifically, our participants mischaracterized the applicability of Bernoulli's equation and the physical meanings of the variables therein.

Misinterpretation of Bernoulli's equation. The fundamental message conveyed by Bernoulli's equation is the constant nature of the sum of three physical terms in an ideal fluid: pressure $(P)$, kinetic energy per unit volume $\left(\frac{1}{2} \rho v^{2}\right)$, and gravitational potential energy per unit volume $(\rho g h)$. It is a mathematical description of a state in hydrodynamics at any instant and can be applied to any points along a streamline. However, some of our teachers misconstrued the ontological nature of Bernoulli's equation and viewed it as a process where changes in the three quantities would not be in synchrony but rather some would lag behind others. For example, one teacher plotted a graph as shown in Fig. 5 to illustrate the relationships between $P$, $\frac{1}{2} \rho v^{2}$, and $\rho g h$. He then explained his work as follows:

T11: Umm... P and $\frac{1}{2} \rho v^{2}$ are different. The pressure $P$ is the state at a certain point in space, which represents the force on the fluid element, while $\frac{1}{2} \rho v^{2}$ is the kinetic energy component, which is gradually acquired or lost by matter...The change of kinetic energy requires work, and there will be no work without distance...Therefore, the change of the kinetic energy component should lag behind $P$ with a distance delay of $x$ (marked in Fig. 5), so there is a distance between force and energy.

Interestingly, the participant drew on the work-energy theorem as a basis to conceptualize a causal relationship between $P$ and $\frac{1}{2} \rho v^{2}$, contending that it was the work done by the force (related to $P$ ) that led to a change in the water's

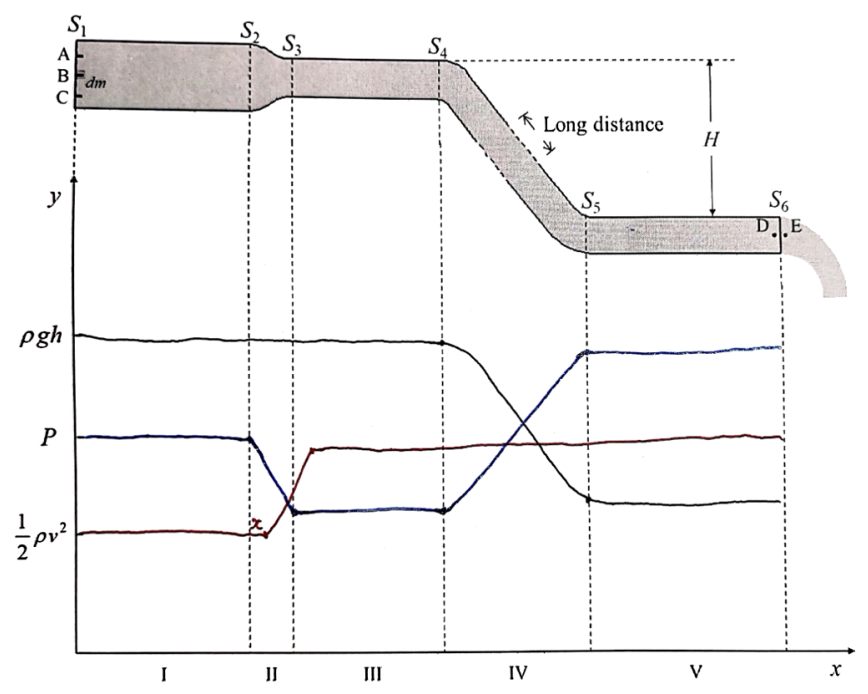

FIG. 5. A graph of $\frac{1}{2} \rho v^{2}, \rho g h$, and $P$ as a function of horizontal position $x$ drawn by a teacher participant (T11).

kinetic energy (related to $\frac{1}{2} \rho v^{2}$ ). As such, he deliberately created a delay (marked as $x$ ) in region II to show the cause slightly preceding the effect over a short distance.

This exact viewpoint was also expressed by a second teacher interviewee, who offered yet another quite revealing explanation when being asked by the interviewer if such a delay would produce any inconsistency with Bernoulli's equation.

Interviewer: Does the result [delay] contradict Bernoulli's equation?

T15: It is not contradictory, because to increase the kinetic energy of the water you need to do work, it is done over a distance and needs time. The final stable result will still be a constant like before. For example, if you pick three elements in Regions I, III and IV respectively, the sums of the three terms in Bernoulli's equation will still be equal for the three regions.

Interviewer: What does "stable" mean?

T15: That's to say regions with a constant velocity.

Here, the participant referred to the non-accelerating water as a stable system and thought it was only under this condition that Bernoulli's equation would apply. Conceivably, this participant mistakenly equated a steady flow to a non-accelerating fluid system, thereby distorting the usage of Bernoulli's equation. It is worth pointing out that although prior studies have documented student difficulties with Bernoulli's equation, there has been no report of ontological mischaracterizations of the nature and applicability of this principle.

Misinterpretation of the quantities in Bernoulli's equation. Besides the aforementioned views about the nature of Bernoulli's equation, some teachers also assigned incorrect meanings to the quantitates in the equation. 
The term of pressure, for example, is such a frequently misunderstood quantity. When using Bernoulli's equation to answer Q3, one teacher responded that water pressure at the outlet of the tube would be greater than atmospheric pressure and provided the following reasoning:

T1: If you place a hand near the outlet of the tube, into the stream, you will feel a strong force, so the water pressure is huge, surely greater than the atmospheric pressure. Umm, like a firehose, it is even more powerful... Also, when the water first flows out, it's a water jet in the shape of a column, and its pressure is very high... Then gradually the column shape of the water jet no longer holds, so the pressure should decrease accordingly... When the water eventually disperses into water drops, it's the same as the atmospheric pressure.

Here, it is important to differentiate two fundamentally different concepts, static pressure $P$ as used in Bernoulli's equation and stagnation pressure (or total pressure) often expressed as the sum of static pressure $P$ and dynamic pressure $\frac{1}{2} \rho v^{2}$. What the above teacher participant referred to, in fact, is a stagnation pressure, not a static pressure denoted by $P$ in Bernoulli's equation. This is because in the example raised by the participant, the water flow was intercepted by a hand. Therefore, the pressure on the hand ought to include both the static component $P$ and the dynamic component $\frac{1}{2} \rho v^{2}$. However, the participant confused the two and ascribed what should be stagnation pressure to the term $P$, thereby reaching an incorrect inference about water pressure at the outlet of the tube.

\section{DISCUSSION AND IMPLICATIONS}

As evidenced in the above findings, the underlying notions that our novice teachers held about hydrodynamics touched upon some of the most fundamental features of fluid dynamics, relating directly to the ontological properties of ideal fluids, the function of force-motion and workenergy analysis, and the nature of Bernoulli's equation. It was on these critical matters that our teachers struggled a great deal, and consequently their responses to the various tasks of the sequential problem revealed a number of errors.

It is interesting and also somewhat surprising to see that our in-service teachers used what should have been the properties of solids and gases to describe an ideal incompressible fluid (water). They confused incompressibility (cf. ideal fluid) with nondeformability (cf. rigid body), assumed the same level of fluidity for liquid and gas by ignoring water cohesion, and conflated the different pressure mechanisms for water and gas respectively. It is worth noting that none of these findings has been systematically reported in the previous literature.

When conducting force-motion and work-energy analysis of fluid elements, our novice teachers could not seem to grasp the meaning of interactions between matter and hence faltered on the basic functions of choosing and isolating a proper system. They often mistook an individual force for a net force or mistook an individual work for a total work to reason about pressure. What was striking here is that they tended to draw on a subsequent event (a downstream flow) as a sole determinant to conclude about a preceding event (an upstream flow), bypassing the needed analysis of the interactions between fluid elements.

As for Bernoulli's equation, which by nature is a description of an instantaneous state of an ideal fluid system, our teachers mischaracterized it as a process and imagined an asynchrony between the different quantities in Bernoulli's equation. What's more, they misconstrued the applicability of the principle by confusing a steady flow with a nonaccelerating system. As a result, they incorrectly stated that Bernoulli's equation could only apply to a system of zero acceleration. These findings are particularly interesting not only because they have not been documented in the previous literature, but also because they speak directly to our teachers' ontological making of the most fundamental principle in hydrodynamics. It is also interesting to see from the study that the teachers often misinterpreted the quantities in Bernoulli's equation and assigned them incorrect meanings.

Besides the above findings, one unique aspect of our study worth highlighting is the use of a sequential synthesis problem. Previous studies of this topic have all relied on employing multiple-choice questions or short-scenario problems [56] to explore learner's misconceptions. Differing from this convention, the problem we designed and administered in the present study contained a set of phenomena or events depicting water undergoing different conditions. By doing so, we obtained several interesting but previously unreported findings. For instance, as mentioned above, the sequenced, continuity feature of the sequential problem allowed us to detect such a tendency that some teachers based their reasoning of an upstream flow solely on the conditions of a downstream flow, neglecting the necessary analysis of a proper system altogether. This pattern of reasoning would not have been uncovered had we used a traditional short-scenario problem. Additionally, the use of multiple representations for the sequenced events helped to reveal otherwise hidden notions of our teachers about Bernoulli's equation. The added delay in the teachers' graphs of $P$ in relation to $\frac{1}{2} \rho v^{2}$ is such an example. Here, by triangulating the findings from the graphs and interviews, we were able to infer deep ontological viewpoints of our teachers about Bernoulli's equation. The graphical representation of the synthesis task further afforded us with a continuous, global view of the ways our teachers determined the three variables along the entire tube. This cannot be achieved by simply piecing together traditional questions that each only asks participants to perform one pairwise comparison at a time. To a large extent, it is similar to the Gestalt effect [57], or simply put, a whole is always greater than, and hence different from, the 
sum of its constituent parts. Perhaps more importantly, the continuous, global plot derived directly from the sequential synthesis task can be useful for teaching Bernoulli's equation. It allows learners to explore the relationships between the three variables at any point along the tube, thereby solidifying the idea that the sum of them is a constant along the entire streamline at any time. This can be conducive to establishing a correct view of Bernoulli's equation as a description of a state rather than a process.

Also important to note is that in this study we selected novice physics teachers as our subjects of interest, an underresearched group in this area. Because of their relatively short time in service, they likely were still experiencing a transition from university students to school teachers and were perhaps grappling with different ways to teach effectively. As seen from our interviews, when reasoning about the properties and motions of an ideal fluid, these teachers often drew on analogies to everyday objects and phenomena to make sense, such as sand in an hourglass, earth moving around the sun, and a water jet from a firehose. While commendable, proper use of analogies is not an easy task. If not carefully constructed, analogies not only will fail to serve the purpose of a model but may also introduce or even reinforce incorrect conceptions. As revealed in our study, the analogies used by our teachers lacked the necessary rigor to help draw useful inferences. Instead, they led our teachers to a mismatch of ontologically different categories of solids, liquids, and gases. To that end, it is critical that we help novice teachers increase their awareness of careful use of proper analogies in classroom teaching.

Finally, a few useful extensions from our current study may be worth pointing out. First, the identified underlying notions of our teachers about hydrodynamics in some sense can be summarized into ontological views of matter, interactions, and energy in ideal fluids. To that end, our study implies that teaching and learning of hydrodynamics requires deliberate attention on highlighting and substantiating these fundamental ideas. Prior studies have shown that engaging learners in peer discussion can be an effective approach to teaching such fundamentals in physics [58-64]. However, successful implementation of peer discussion requires research-based instructional materials that can maximize students' active participation. Without such resources, peer discussion can hardly be effective. As part of our on-going efforts to implement peer discussion and to evaluate its effectiveness in teacher education [61,65-68], our studies of learners', particularly teachers' conceptions of physics topics are an important step forward.

In this current study, although we have captured a set of key notions about hydrodynamics held by our novice teachers, we have no definitive answer regarding the extent to which our findings may be applied to all populations of secondary physics teachers. That said, our findings still hold significant merits, and the validity thereof still stands. As illustrated above, the sequential synthesis problem we used in the study contains sequenced events or phenomena, each representing a scenario that requires application of the same fundamental concepts but varies in surface features and representation formats. To that end, one synthesis problem, in effect, contains multiple "isomorphic" subproblems $[69,70]$. By using these varying scenarios, we effectively captured the teachers' errors in applying Bernoulli's equation, some of which are being reported for the first time. Additionally, the interviews we conducted in the study solicited not only the participants' thoughts on the task but also other relevant ideas they encountered elsewhere. This is exactly how we were able to capture from the interviews the participants' use of metaphors and references to everyday experience, such as hourglass and firehose. In other words, these findings are not idiosyncratic, but rather they have a base in the participants' prior experiences. Further, it is important to note that our study is not an experiment or a quasiexperiment, in which one can rightly examine the relationships between participants' demographics and their problem-solving performances in the hopes of generalizing findings across all populations [71]. Instead, this is an exploratory study aimed at using a sequential synthesis task to detect teachers' understandings of fluid dynamics. We must be cautious in choosing proper criteria to judge the merits of research findings. Regardless, more investigations along this line of work are worth pursuing.

\section{APPENDIX: CORE CONCEPTS IN FLUID DYNAMICS AND ANSWERS TO SEQUENTIAL SYNTHESIS PROBLEM}

1. Below is a list of fluid dynamics concepts relevant to Bernoulli's equation that are required of undergraduate physics majors in China.

\begin{tabular}{ll} 
Streamline & Streamlines are curves tangential to the velocity vectors of fluid elements at an instant of time. \\
\hline $\begin{array}{l}\text { Path line } \\
\text { Steady flow }\end{array}$ & $\begin{array}{l}\text { Path lines are trajectories of fluid elements over a period of time. } \\
\text { A steady flow means a flow that is steady with respect to time. Thus, all conditions of the flow at any } \\
\text { point remain constant with respect to time. } \\
\text { An ideal fluid is a fluid in which there is no friction. It is inviscid (zero viscosity). }\end{array}$ \\
\hline
\end{tabular}

(Table continued) 
(Continued)

Incompressible fluid

Continuity equation

Bernoulli's equation

Static pressure

Stagnation pressure
Fluids that maintain a constant density. Liquids are relatively incompressible, so we can approximate them as wholly incompressible.

$S_{1} v_{1}=S_{2} v_{2}$

$S_{1}$ and $S_{2}$ : cross sections at locations 1 and 2, respectively

$v_{1}$ and $v_{2}$ : speeds of a flow at locations 1 and 2 , respectively

The continuity equation is applicable to any incompressible fluids, be they a steady or a nonsteady flow.

$P+\rho g h+\frac{1}{2} \rho v^{2}=$ const

$P$ : pressure (or more precisely static pressure)

$\rho$ : density

$h$ : height

$v$ : speed

$g$ : gravitational acceleration

Assumptions:

1. Zero viscosity (no friction)

2. Steady, streamline flow

3. Applicable to any points along a streamline in an incompressible fluid

4. No energy change in the fluid flow along the same streamline

Static pressure $P$ as denoted in Bernoulli's equation is the pressure that an instrument could measure if it were static with respect to the fluid, i.e., moving with the fluid.

Devices, such as piezometer tubes, can be used to measure static pressure.

Stagnation pressure, also known as total pressure, is the sum of static pressure $(P)$ and dynamic pressure $\left(\frac{1}{2} \rho v^{2}\right)$. It is the pressure at a point where the fluid comes to rest.
2. Answers to the sequential synthesis problem.

Q1

See Fig. 6.

Q2

$v$ and $P$ remain constant along region $\mathrm{I}$.

$v$ increases and $P$ decreases along region II.

$v$ and $P$ remain constant along region III. $v$ remains constant and $P$ increases along region IV. $v$ and $P$ remain constant along region $\mathrm{V}$.

Q3

$v_{D}=v_{E}$

$P_{D}=P_{E}=P_{0}$

Q4

See Fig. 7.

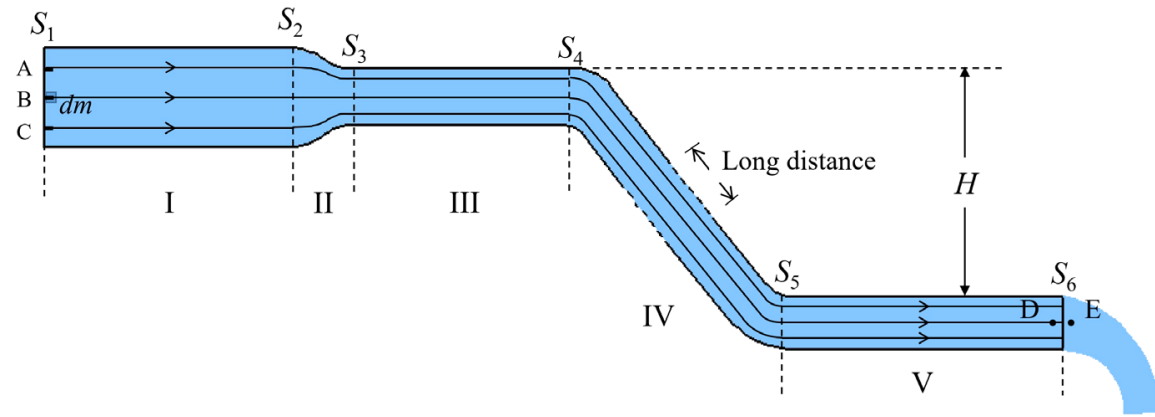

FIG. 6. Streamlines of the water flow in different regions. 


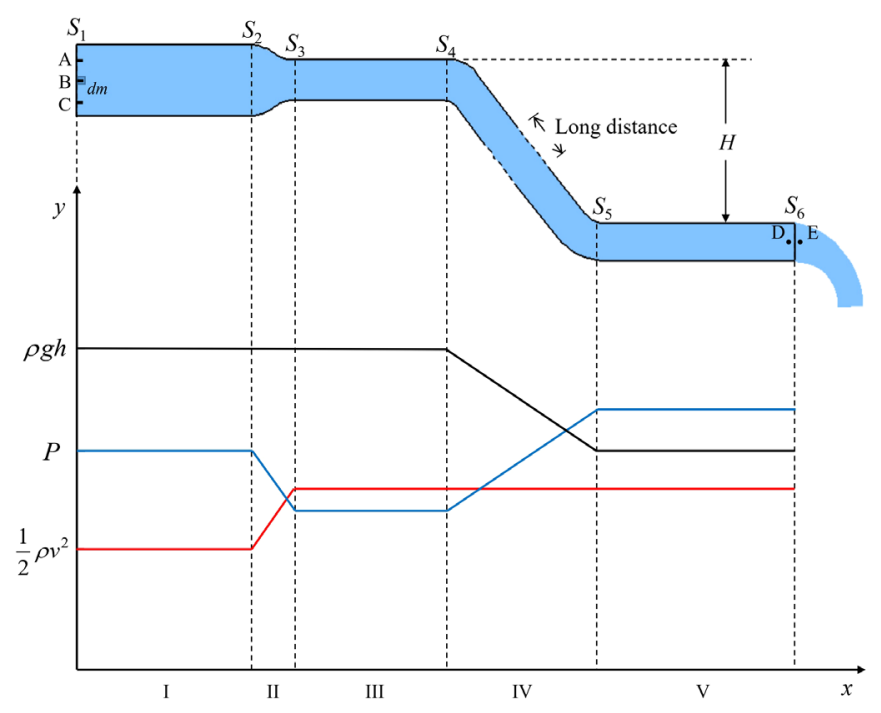

FIG. 7. Graphical representation of $P, \rho g h$, and $\frac{1}{2} \rho v^{2}$ in different regions.

[1] M. Gad-El-Hak, Fluid mechanics from the beginning to the third millennium, Int. J. Engng Ed. 14, 177 (1998), https:// www.ijee.ie/articles/Vol14-3/ijee1019.pdf.

[2] Q. Peng,物理（八年级下) [Physics (Grade 8, Volume 2)] (People's Education Press, Beijing, 2012).

[3] J. L. Docktor and J. P. Mestre, Synthesis of disciplinebased education research in physics, Phys. Rev. ST Phys. Educ. Res. 10, 020119 (2014).

[4] L. C. McDermott and E. F. Redish, Resource letter: PER-1: Physics education research, Am. J. Phys. 67, 755 (1999).

[5] M. Goszewski, A. Moyer, Z. Bazan, and D. J. Wagner, Exploring student difficulties with pressure in a fluid, AIP Conf. Proc. 1513, 154 (2013).

[6] U. Besson, Students' conceptions of fluids, Int. J. Sci. Educ. 26, 1683 (2004).

[7] M. E. Loverude, C. H. Kautz, and P. R. L. Heron, Helping students develop an understanding of Archimedes' principle. I. Research on student understanding, Am. J. Phys. 71, 1178 (2003).

[8] P. R. L. Heron, M. E. Loverude, P. S. Shaffer, and L. C. McDermott, Helping students develop an understanding of Archimedes' principle. II. Development of research-based instructional materials, Am. J. Phys. 71, 1188 (2003).

[9] M. E. Loverude, P. R. L. Heron, and C. H. Kautz, Identifying and addressing student difficulties with hydrostatics pressure, Am. J. Phys. 78, 75 (2010).

[10] P. Kariotoglou and D. Psillos, Pupils' pressure models and their implications for instruction, Res. Sci. Technol. Educ. 11, 95 (1993).

[11] P. Kariotogloy, D. Psillos, and O. Vallassiades, Understanding pressure: didactical transpositions and pupils' conceptions, Phys. Educ. 25, 92 (1990).
[12] E. E. Glough and R. Driver, What do children understand about pressure in fluids?, Res. Sci. Technol. Educ. 3, 133 (1985).

[13] O. A. Dentici, M. G. Grossi, L. Borghi, A. D. Ambrosis, and C. I. Massara, Understanding floating: a study of children aged between six and eight years, Int. J. Sci. Educ. 6, 235 (1984).

[14] O. Saputra, A. Setiawan, and D. Rusdiana, Identification of student misconception about static fluid, J. Phys. Conf. Ser. 1157, 032069 (2019).

[15] K. Y. Manoj, Clarifying the misconception about the principle of floatation, Phys. Educ. 49, 523 (2014).

[16] J. K. Martin, J. Mitchell, and T. Newell, Work in progress: analysis of reliability of the fluid mechanics concept inventory, in Proceedings of the Frontiers in Education Conference 2004 (IEEE, Savannah, GA, 2004), p. F1F-3.

[17] J. Martin, J. Mitchell, and T. Newell, Development of a concept inventory for fluid mechanics, in Proceedings of the Frontiers in Education Conference 2003 (IEEE, Boulder, CO, 2003), p. T3D-23.

[18] M. K. Watson, A. R. Mills, K. C. Bower, K. Brannan, M. H. Woo, and R. W. Welch, Refinement of a concept inventory to assess conceptual understanding in civil engineering fluid mechanics, in Proceedings of the 122nd ASEE (American Society for Engineering Education, Washington DC, 2015).

[19] P. Eastwell, Bernoulli? Perhaps, but what about viscosity?, Sci. Educ. Rev. 6, 1 (2007), https://eric.ed .gov/?id=EJ1050910.

[20] M. Kamela, Thinking about Bernoulli, Phys. Teach. 45, 379 (2007).

[21] P. Eastwell, Thinking some more about Bernoulli, Phys. Teach. 46, 134 (2008). 
[22] N. F. Smith, Bernoulli and Newton in fluid mechanics, Phys. Teach. 10, 451 (1972).

[23] R. P. Bauman and R. Schwaneberg, Interpretation of Bernoulli's equation, Phys. Teach. 32, 478 (1994).

[24] A. B. Murphy and S. Brusca, Bernoulli effect, Phys. Educ. 21, 262 (1986).

[25] K. Weltner, Misinterpretations of Bernoulli's law, http:// user.uni-frankfurt.de/ weltner (2011).

[26] C. Swartz, Bernoulli and Newton, Phys. Teach. 41, 196 (2003).

[27] D. G. F. Huilier, Forty years' experience in teaching fluid mechanics at Strasbourg University, Fluids 4, 199 (2019).

[28] D. M. Kurniawati and F. U. Ermawati, Analysis students' conception using four-tier diagnostic test for dynamic fluid concepts, J. Phys. Conf. Ser. 1491, 012012 (2020).

[29] D. M. Fraser, R. Pillay, L. Tjatindi, and J. M. Case, Enhancing the learning of fluid mechanics using computer simulations, J. Eng. Educ. 96, 381 (2007).

[30] D. H. Martin, Misunderstanding Bernoulli, Phys. Teach. 21, 37 (1983).

[31] A. Suarez, S. Kahan, G. Zavala, and A. C. Marti, Student conceptual difficulties in hydrodynamics, Phys. Rev. Phys. Educ. Res. 13, 020132 (2017).

[32] S. Brown, K. Beddoes, D. Montfort, and A. Baghdanov, Engineering students' fluid mechanics misconceptions: A description and theoretical explanation, Int. J. Eng. Educ. 33, 1149 (2017), https://dialnet.unirioja.es/servlet/articulo? codigo $=6897037$.

[33] J. D. Anderson, Fundamentals of Aerodynamics (McGrawHill, New York, 1984).

[34] L. D. Landau and E. M. Lifshitz, Fluid Mechanics, 2nd ed. (Pergamon Press, New York, 1987).

[35] E. J. Finnemore and J. B. Franzini, Fluid Mechanics with Engineering Applications, 10th ed. (McGraw-Hill, New York, 2012).

[36] A. Qi and C. Du, A Course in General Physics: Mechanics, 2nd ed. (Higher Education Press, Beijing, 2005).

[37] G. W. Recktenwald, R. C. Edwards, D. Howe, and J. Faulkner, A simple experiment to expose misconceptions about the Bernoulli's equation, in Proceedings of the ASME International Mechanical Engineering Congress and Exposition (American Society of Mechanical Engineers, New York, 2009).

[38] M. T.H. Chi, Commonsense conceptions of emergent processes: Why some misconceptions are robust, J. Learn. Sci. 14, 161 (2005).

[39] M. T. H. Chi, Three types of conceptual change: Belief revision, mental model transformation, and categorical shift, in Handbook of Research on Conceptual Change, edited by S. Vosniadou (Routledge, New York, 2008), p. 61.

[40] J. Hoehn, J. Gifford, and N. Finkelstein, Investigating the dynamics of ontological reasoning across contexts in quantum physics, Phys. Rev. Phys. Educ. Res. 15, 010124 (2019).

[41] R. Scherr, H. Close, S. McKagan, and S. Vokos, Representing energy. I. Representing a substance ontology for energy, Phys. Rev. Phys. Educ. Res. 8, 020114 (2012).

[42] B. Dreyfus, B. Geller, J. Gouvea, V. Sawtelle, C. Turpen, and E. Redish, Ontological metaphors for negative energy in an interdisciplinary context, Phys. Rev. Phys. Educ. Res. 10, 020108 (2014).

[43] S. Vosniadou, Capturing and modeling the process of conceptual change, Learn. Instr. 4, 45 (1994).

[44] S. Vosniadou, The cognitive-situative divide and the problem of conceptual change, Educ. Psychol. 42, 55 (2007).

[45] A. A. diSessa, Toward an epistemology of physics, Cognit. Instr. 10, 105 (1993).

[46] D. Hammer, Student resources for learning introductory physics, Am. J. Phys. 68, S52 (2000).

[47] D. E. Meltzer, Relation between students' problem solving performance and representational format, Am. J. Phys. 73, 463 (2005).

[48] P. B. Kohl and N. D. Finkelstein, Student representational competence and self-assessment when solving problems, Phys. Rev. ST Phys. Educ. Res. 1, 010104 (2005).

[49] Y. Cao and B. M. Brizuela, High school students' representations and understandings of electric fields, Phys. Rev. Phys. Educ. Res. 12, 020102 (2016).

[50] B. Ibrahim, L. Ding, A. F. Heckler, D. R. White, and R. Badeau, Students' conceptual performance on synthesis physics problems with varying mathematical complexity, Phys. Rev. Phys. Educ. Res. 13, 010133 (2017).

[51] L. Ding, N. Reay, A. Lee, and L. Bao, Exploring the role of conceptual scaffolding in solving synthesis problems, Phys. Rev. Phys. Educ. Res. 7, 020109 (2011).

[52] L. Ding, N. W. Reay, A. Lee, and L. Bao, Using conceptual scaffolding to foster effective problem solving, AIP Conf. Proc. 1179, 129 (2009).

[53] L. Ding, N. W. Reay, A. F. Heckler, and L. Bao, Sustained effects of solving conceptually scaffolded synthesis problems, AIP Conf. Proc. 1289, 133 (2010).

[54] B. Ibrahim, L. Ding, A. F. Heckler, D. R. White, and R. Badeau, How students process equations in solving quantitative synthesis problems? Role of mathematical complexity in students' mathematical performance, Phys. Rev. Phys. Educ. Res. 13, 020120 (2017).

[55] B. Ibrahim and L. Ding, Sequential and simultaneous synthesis problem solving: A comparison of students' gaze transitions, Phys. Rev. Phys. Educ. Res. 17, 010126 (2021).

[56] A Lee, L. Ding, N. W. Reay, and L. Bao, Single-concept clicker question sequences, Phys. Teach. 49, 385 (2011).

[57] K. Koffka, Principles of Gestalt Psychology (Kegan Paul, Trench, Trubner \& Co., Ltd., London, 1936).

[58] R. R. Hake, Interactive-engagement versus traditional methods: A six-thousand-student survey of mechanics test data for introductory physics courses, Am. J. Phys. 66, 64 (1998).

[59] P. Zhang, Q. Y. Tu, and Y. P. Mo, 课堂中的合作学习同伴教学法对物理概念学习的促进作用 [Collaborative learning in classroom - Implementing peer instruction to promote learning of physics concepts], 中国大学教学 [China University Teaching] 6, 56 (2012).

[60] R. Leinonen, M. A. Asikainen, and P. E. Hirvonen, Overcoming students' misconceptions concerning thermal physics with the aid of hints and peer interaction during a lecture course, Phys. Rev. Phys. Educ. Res. 9, 020112 (2013). 
[61] P. Zhang, L. Ding, and E. Mazur, Peer Instruction in introductory physics: A method to bring about positive changes in students' attitudes and beliefs, Phys. Rev. Phys. Educ. Res. 13, 010104 (2017).

[62] C. Singh, Impact of peer interaction on conceptual test performance, Am. J. Phys. 73, 446 (2005).

[63] B. Bungum, M. V. Bøe, and E. K. Henriksen, Quantum talk: How small-group discussions may enhance students' understanding in quantum physics, Sci. Educ. 102, 856 (2018).

[64] A. Samsudin, N. Fratiwi, and N. Amin, Wiendartun, Supriyatman, F. Wibowo, M. Faizin, and B. Costu, Improving students' conceptions on fluid dynamics through peer teaching model with PDEODE (PTMPDEODE), J. Phys. Conf. Ser. 1013, 012040 (2018).

[65] P. Zhang, 基于翻转课堂的同伴教学法: 原理 方法 实践 [Flipped Classroom-Peer Instruction: Principle and Practice] (Post \& Telecom Press, Beijing, 2017).

[66] P. Zhang, L. Ding, and W. S. Zhang, 翻转课堂的理念、 演变与有效性研究 [Flipped classroom: Theory, development history and effectiveness], 教育学报 [J. Educ. Studies] 13, 46 (2017).
[67] P. Zhang, W. Qi, Y. P. Wang, and Q. Q. Zhang, 基于 Peer Instruction 教学方法研究大学生物理错误概念一以动 生电动势为例 [Detecting the undergraduates' misconceptions of physics using Peer Instruction-The case of the motional electromotive force], 物理与工程 [Phys. Eng.] 24, 56 (2014).

[68] P. Zhang and Y. Y. Liu, 同伴教学法在大学物理课程中的 应用 [Using Peer Instruction to teach an introductory physics], 物理与工程 [Phys. Eng.] 22, 41 (2012).

[69] L. Ding and N. W. Reay, Teaching undergraduate introductory physics with an innovative research-based clicker methodology, in Research Based Undergraduate Science Teaching, edited by D. Sunal, C. Sunal, E. Wright, C. Mason, and D. Zollman (Information Age Publishing, Charlotte, NC, 2014), Vol. 6, p. 301.

[70] L. Ding, N. W. Reay, A. Lee, and L. Bao, Are we asking the right questions? Validating clicker question sequences by student interviews, Am. J. Phys. 77, 643 (2009).

[71] L. Ding, Theoretical perspectives of quantitative physics education research, Phys. Rev. Phys. Educ. Res. 15, 020101 (2019). 